\title{
45 Cases of Surgical Treatment of Ovarian Cancer and Analysis on Effect of Intraperitoneal Chemotherapy
}

\author{
Bao Lihong ${ }^{1}$, Sa Qila ${ }^{* 2}$, Sun Ping ${ }^{2}$, Wang Yukun ${ }^{3}$
}

1. Medical College, Inner Mongolia University for the Nationalities, Tongliao, Inner Mongolia, 028000, China

2. Acadamy of Mongolian Medicine, Inner Mongolia Medical University; Inner Mongolia, Huhhot, 010110, China

3. Department of Obstetrics and Gynecology, Affiliated Hospital of National University of Inner Mongolia, Tongliao, Inner Mongolia, 028000,China

\begin{abstract}
Objective: This study puts its focus on the factors that affect the prognosis of recurrent ovarian cancer, so as to explore the factors of improving the therapeutic effect and prolong the survival time of patients. Method: Making retrospective analysis on 45 cases of having complete clinical treatment with recurrent ovarian cancer in Affiliated Hospital of Medical College of Inner Mongolia University from 2010 to 2011, completing the survey and classification and statistics according to age, residual tumor size, pathological type, number of chemotherapy period. single factor analysis on COX proportional hazards regression model by adopting Pearson correlation analysis is made. Result: Study reults show that the time of progression free survival (PFS) is related to the time of the overall survival (OS), the longer PFS is, the longer OS is. $(\mathrm{P}<0.01)$. Moreover, the primary surgical treatment of residual tumor size, number of chemotherapy period, and stage of disease can all affect OS of the recurrent ovarian cancer. $(\mathrm{P}<0.05)$. Conclusion: This study puts its focus on the factors that affect the prognosis of recurrent ovarian cancer, so as to explore the factors of improving the therapeutic effect and prolong the survival time of patients. Thus it can provide reference for better improving the clinical treatment effect of recurrent ovarian cancer patients.
\end{abstract}

Keywords: Ovarian cancer, Progression free survival, Prognosis of recurrent

\section{Introduction}

The incidence of ovarian cancer accounted for the third place in the gynecological malignant tumor, but the mortality rate is the highest. According to the statistics from U.S. Centers for Disease Control in 2010, 20180 women are diagnosed with ovarian cancer in the U.S. in 2010, among them, 15310 women died of ovarian cancer [1]. Due to the lack of specific symptoms and signs in the early stage of the ovary, and the lack of an effective screening strategy, it is difficult to diagnose it early in the early stage of the disease, so about $70 \%$ of the patients have been found to be advanced. The treatment effect of patients with late stage is poor, and the five-year survival rate hovers around $30 \%$ [2]. At present, the standard treatment for the advanced ovarian cancer is cytoreductive surgery and the chemotherapy based on platinum[3]. In recent years, with the development of surgery and chemotherapy, the response rate of first-line chemotherapy is as high as $70-80 \%$, and the survival rate of ovarian cancer has been improved. However, due to the characteristics of drug resistance and recurrence, the vast majority of patients will eventually relapse, who need to find out non-platinum agent or biological therapy method. Once the recurrence of ovarian cancer occurred, the treatment 
principle is to palliative rather than cure, the treatment aims to relieve symptoms, so as to improve the quality of life, as far as possible to prolong PFS and OS of patients. This study mainly puts its focus on the factors that affect the prognosis of recurrent ovarian cancer, so as to improve the therapeutic effect and prolong the survival time of patients

\section{Material and Method}

\section{General Information}

We collected 45 cases of patients with recurrent ovarian cancer in Obstetrics and Gynecology Hospital of Inner Mongolia University from August 2010 to June 2011and completed follow-up (including 7 cases of survival), the deadline for follow-up is April 2016, their first operations were performed in our hospital. The youngest patient was 22 years old, while the oldest patient was 74 years old, the average age was 50 years old.

\section{Pathological Type}

There were 29 cases of serous carcinoma (64.4\%), 1 case of mucinous carcinoma (2.2\%), 4 cases of endometrioid adenocarcinoma (8.8\%), 11 cases of poorly differentiated adenocarcinoma (24.4\%).

\section{Surgical Staging}

According to the method of FIGO2000 staging, among then,3 cases in stage I (6.7\%), 7 cases in stage II (15.5\%), 28 cases in stage III (62.2\%), 7cases in stage IV (15.5\%).

\section{Surgical Method}

In the early stage (stage I and stage II), stage operation for ovarian carcinoma is carried out, while in the late stage (stage III and IV), cytoreductive surgery is carried out, among them, 21 cases of having the largest residual tumor, which is $>2 \mathrm{~cm}$ (46.7), 23 cases of having the largest residual tumor, which is $<2 \mathrm{~cm}(53.3 \%)$.

\section{Chemotherapy Method}

All the patients were treated with chemotherapy, which is based on platinum, among them, 36 cases of having treatment period, which is $>6(80 \%)$ and 9 cases of having treatment period, which is $<6(20 \%)$.

\section{Statistical Method}

SPSS17.0 software was adopted, the PFS influencing factors can be analyzed by COX proportional hazards regression model. The relationship between PFS and OS can be analyzed by Pearson correlation analysis; $\mathrm{P}<0.05$ is statistically significant.

\section{Result}

PFS and OS: among 45 patients, PFS is from 4 month to 101 months, the average PFS is 11 months. Among these followed up patients, 5 cases of patients survived, 40 cases of patients died, among the death cases, OS is 1875 months, the average OS is 43.5 months.

The survival rate of patients for one year, three-year and five-year is $86.5 \%, 71.2 \%$, and $36.5 \%$ respectively.

The relationship between PFS and the survival time in the 45 cases of death, compared with the time of PFS and OS after the first treatment, we can find out that there is a positive correlation between them, after the first treatment, the longer PFS is, the longer OS is $(\mathrm{P}<0.01)$.

The relevant factors that affect OS among these 45 patients, including age, pathological type, clinical stage, postoperative residual tumor size, as well as the number of having chemotherapy can carry on multivariate analysis, the results showed us that age, pathological type had no significant correlation with OS ( $\mathrm{P}>0.05)$, while clinical stage, residual tumor size, number of period of treatment 
had significant correlation with OS $(\mathrm{P}<0.05)$. The earlier the clinical stage is, the smaller the residue size is, the longer OS is. (Table 1)

Table 1 The Effect of Different Factors on Ovarian Cancer OS

\begin{tabular}{ccccccc}
\hline & $\begin{array}{c}\text { Regression } \\
\text { coefficient (B) }\end{array}$ & $\begin{array}{c}\text { Standard error } \\
\text { (S.E.) }\end{array}$ & Wald & df & $\begin{array}{c}\text { Significan } \\
\text { t level (P) }\end{array}$ & $\begin{array}{c}\text { Exp } \\
\text { (B) }\end{array}$ \\
\hline $\begin{array}{c}\text { Pathological } \\
\text { type }\end{array}$ & -0.057 & 0.134 & 0.184 & 1 & 0.668 & 0.944 \\
$\begin{array}{c}\text { Residual } \\
\text { lesion }\end{array}$ & 1.141 & 0.334 & 11.681 & 1 & 0.001 & 3.129 \\
$\begin{array}{c}\text { Stage } \\
\text { Period of } \\
\text { treatment }\end{array}$ & 0.499 & 0.240 & 4.338 & 1 & 0.037 & 1.647 \\
\hline
\end{tabular}

\section{Discussion}

\section{Prognostic Factors of Affecting Recurrent Ovarian Cancer}

The major factors that can affect the recurrent ovarian cancer can be including clinical stage, pathological type, residual tumor size, degree of differentiation, postoperative chemotherapy, as well as the patient's physical condition. The clinical stage is an important factor that can affect the recurrent ovarian cancer, the earlier FIGO staging is, the lower recurrent rate the patient has. While most of patients in the advanced or late stage may have recurrent ovarian cancer in stage I or stage II, the epithelial ovarian cancer of different pathological types, with different malignant degree can have different effect on recurrence, serous carcinoma, clear cell carcinoma mucinous carcinoma can be easily have the recurrence[4-5]. Differentiation degree of cancer cells determines the speed of cell division and transfer capabilities, the lower degree of cell differentiation can be easily have the recurrence. The postoperative residual tumor size can have direct effect on tumor cell sensitivity to chemotherapy, when the residual tumor diameter is greater than $2 \mathrm{~cm}$, it can be easily have the recurrence. Large clinical studies have found that the recurrent rate of choosing chemotherapy regimens based on non-platinum is significantly higher than that of it based on platinum. The patients with poor physical condition, old age or with other complication symptoms can easily have the recurrence that people with young age without complication symptoms.

\section{Treatment of Recurrent Ovarian Cancer}

\section{Principle of Treatment}

The treatment of recurrent ovarian cancer should take these factors into account, such as the wishes of patients, treatment effect, treatment costs and quality of life, and so on, so as to choose suitable treatment plan. Due to the lack of large sample prospective randomized controlled clinical trial (RCT) data, it can not prove which program or the preparation have the positive effect, therefore, so far ROC has no standard treatment plan, there is no better treatment. At present, salvage chemotherapy and two cytoreductive surgery is widely used in the field of treatment. It is almost impossible to cure recurrent ovarian cancer. The main treatment is palliative chemotherapy, which can be regarded as salvage chemotherapy. The purpose of the treatment is to alleviate the symptoms caused by the tumor, so as to prolong the life and remission period and reduce the side effects of treatment, which can ultimately improve the life of patient. 


\section{Conclusion}

According to the clinical observation, PFS of epithelial ovarian cancer patient after having the first time treatment is related to OS of patient with relapse. The longer PFS is, the longer OS will be. Patients with epithelial ovarian cancer in early clinical stage, treatment of postoperative residual tumor size and primary cytoreductive surgery after chemotherapy is related to PFS, the earlier the clinical staging is with postoperative regular full course chemotherapy, the longer PFS will be, the difference of PFS between different pathological types need further clinical studies.

Acknowledgements: Scientific research projects of the Inner Mongolian higher educational system NJZY171; Science research project of Inner Mongolia University for the Nationalities: NMDGP1418.

\section{Reference}

[1] Agarwal, Roshan, and Stan B. Kaye. "Ovarian cancer: strategies for overcoming resistance to chemotherapy." Nature Reviews Cancer 3.7 (2003): 502-516.

[2] Zhao X Y, Huang H F, Lian L J, et al. Ovarian cancer in pregnancy: a clinicopathologic analysis of 22 cases and review of the literature[J]. International Journal of Gynecological Cancer, 2006, 16(1): 8-15.

[3] Young, Robert C., et al. "Adjuvant therapy in stage I and stage II epithelial ovarian cancer: results of two prospective randomized trials." New England Journal of Medicine 322.15 (1990): 1021-1027.

[4] Behtash N, Zarchi M K, Gilani M M, et al. Ovarian carcinoma associated with pregnancy: a clinicopathologic analysis of 23 cases and review of the literature[J]. BMC pregnancy and childbirth, 2008, 8(1): 3.

[5] Su Y. Review of Craniofacial Plastic Correction Method Based on Computer Aided Technology[J]. Journal of Applied Science and Engineering Innovation, 2015, 2(11): 431-434.

[6] Hillner, Bruce E., and Thomas J. Smith. "Efficacy and cost effectiveness of adjuvant chemotherapy in women with node-negative breast cancer: a decision-analysis model." New England Journal of Medicine 324.3 (1991): 160-168.

[7] Pujade-Lauraine, Eric, et al. "Intraperitoneal recombinant interferon gamma in ovarian cancer patients with residual disease at second-look laparotomy."Journal of clinical oncology 14.2 (1996): 343-350. 